\title{
Review
}

\section{Safety, immunogenicity, and efficacy of Borrelia burgdorferi outer surface protein A (OspA) vaccine: A meta-analysis}

\author{
Hua Zhao ${ }^{1,3,4}$, Fukai Frank Bao ${ }^{1,3,4}$ Aihua Liu',3,4 \\ ${ }^{1}$ Department of Microbiology and Immunology, Kunming Medical University, Kunming, China \\ 2 Department of Biochemistry and Molecular Biology, Kunming Medical University, Kunming, China \\ ${ }^{3}$ Yunnan Province Key Laboratory for Tropical Infectious Diseases in Universities, Kunming, China \\ ${ }^{4}$ Yunnan Demonstration Base of International Science and Technology Cooperation for Tropical Diseases, \\ Kunming, China
}

\begin{abstract}
Introduction: Lyme borreliosis, caused by Borrelia burgdorferi sensu stricto in the United States and by several Borrelia species in Europe and Asia, has a great impact on the health of the global population. There are human vaccines available, such as the outer surface protein A (OspA) vaccine, but still more evidence is needed to verify its function. We investigated the safety, immunogenicity, and efficacy of adjuvanted or non-adjuvanted vaccines containing protective epitopes from Borrelia species OspA serotypes in healthy adults.

Methodology: Seven electronic databases were searched for clinical trials involving vaccine of OspA, with outcome data on safety, immunogenicity, and efficacy. The meta-analysis method was used to compare all vaccination strategies at the same time.

Results: Three relevant studies were identified. All were randomized controlled trials (RCTs) or quasi-RCTs. Meta-analysis shows that, compared with low dose, high dose comes with a higher IgG titer with overall effect size of 6.39 . For the $30 \mu \mathrm{g}$ dose, the geometric mean titer was 6918.31, which is statistically significant when compared with 0 . With respect to safety, only soreness showed a relatively high incidence of $40 \%$ ( $\mathrm{p}<0.05$ when compared with 0 , while the other side effects were no difference compared with 0 ).

Conclusions: The OspA vaccine against Lyme disease is safe and its immunogenicity and efficacy have been verified. Instead of stagnating or giving up, further research on improving the vaccine is needed. On the foundation of preliminary studies, we can attempt to develop new vaccines for human use.
\end{abstract}

Key words: vaccine; Lyme disease; OspA; safety; immunogenicity; efficacy

J Infect Dev Ctries 2017; 11(1):1-9. doi:10.3855/jidc.7999

(Received 10 December 2015 - Accepted 11 February 2016)

Copyright $@ 2017$ Zhao et al. This is an open-access article distributed under the Creative Commons Attribution License, which permits unrestricted use, distribution, and reproduction in any medium, provided the original work is properly cited.

\section{Introduction}

Lyme borreliosis, caused by infection with tickborne bacterial spirochetes Borrelia burgdorferi sensu lato species complex [1,2], is a multisystem inflammatory disease. Early skin lesions have an expanding ring form, often with a central clear zone. Fever, chills, myalgia, and headache are common symptoms [3]. Infected individuals can also develop more serious manifestations having impact on the skin (lesions, atrophy, and fibrous nodule formation), nervous system (facial palsy, meningitis, myelitis, and encephalitis), joints (recurrent or persistent large joint synovitis), or heart (conduction abnormalities and carditis) [4].

Lyme borreliosis can be successfully treated with antibiotics [1,2]. Many attempts have been made to develop a vaccine against Lyme disease, and many studies have concentrated on some antigenic subunits of
Borrelia burgdorferi, such as OspA, OspB, OspC, DbpA, and Bbk32(p35). Kolb et al. [5] found that the vaccine from Salp15, which binds to Borrelia burgdorferi outer surface protein $\mathrm{C}(\mathrm{OspC})$, is more effective in preventing Lyme disease. Small et al. [6] found that immunization with peptide B (a peptide derived from Borrelia burgdorferi BB0172 protein) protected mice against Lyme disease. Compared to the other treatment groups, peptide B-immunized mice showed very limited inflammation in the heart and joint tissues. And surprisingly, the anti-peptide B antibodies did not cross-react with $\mathrm{Bb}$ lysates, which suggests that peptide $\mathrm{B}$ is a promising candidate for the development of a new DIVA vaccine (differentiate between infected and vaccinated animals) for protection against Lyme diseases.

However, most of the research on vaccine is focused on outer surface protein A (OspA), with animal 
and human subjects [7-12]. A mouse model of vaccination with recombinant OspA protein was employed in initial research concerning the protection efficacy of OspA [13]. Against a test by intradermal vaccination of refined Borrelia burgdorferi, long-time (180 days) protection was evoked [14]. Needle-infused Borrelia was utilized in this test as an experimental infection model. In 1992, a vector-transmitted infection model was established. At the point when inoculation with OspA was tried in this way, protection efficacy was discovered to be vital, and borreliacidal antibodies from OspA-vaccinated mice could act out of the organisms of the mice and could clear Borrelia in the tick digestive tract preceding their entrance into the inoculated animal [15]. Two large-sample, randomized, double-blind clinical trials in the United States $[16,17]$ showed that Lyme disease could be prevented by vaccination with OspA, but the evidence is still limited and a comprehensive and system evaluation to Lyme vaccine is needed; this was the purpose of our study.

\section{Methodology}

Data sources and searches

Both English and non-English language publications on the Cochrane library, PubMed, Science Direct (Elsevier) Journal, ISI Web of Science, China National Knowledge Internet (CNKI), Wan Fang database, and Sinomed database were searched, with a date range from 1 January 1980 to 30 April 2015. Search terms were "vaccine" and "OspA" or "outer surface protein A" and "Lyme disease" or "Lyme borreliosis".

\section{Study selection}

Criteria were randomized, double-blind clinical trials. Studies were multicenter or single center; threearm or two-arm studies were all included.

For references excluded from the meta-analysis, the criteria were non-human studies, letters/case reports, observational studies, studies including fewer than 10 individuals, articles not reporting outcomes of interest or primary data (editorials, reviews), or using inadequate case definitions. Studies in which the patients had a severe adverse event were excluded.

The main outcome measures were incidence of side effects (local or system), which reflects the safety of the OspA vaccine and IgG antibody titer after injection, which in turn reflects the immunogenicity and efficacy of the OspA vaccine.

\section{Data extraction and quality assessment}

Data were extracted from each study separately by two authors, using predefined inclusion criteria, exclusion criteria, and a data extraction sheet based on the Cochrane Handbook for Systematic Reviews of Intervention. The analysis was carried out in concordance with the Cochrane Handbook of Systematic Reviews and reported based on PRISMA guidelines. The initial agreement between the two reviewers for data selection and data validity assessment of studies was assessed using Kappa coefficient. Discrepancies between the reviewers were resolved by joint discussion and mutual agreement.

\section{Data synthesis and analysis}

The meta-analysis was performed based on findings from individual studies of different researchers. The meta-analyses were conducted using either the randomeffects model or fixed-effect model, decided by a heterogeneity test $[18,19]$. Forest plot and funnel plot were generated to judge the overall effect size and to judge whether there was publication bias, which could be verified by the Egger test [20,21].

Two reviewers independently assessed the quality of the three included studies using the Jadad score [22], which scored based on three aspects: (1) randomization (2-0); (2) blind (2-0); (3) withdrawals and dropouts (10 ). The Jadad score ranged from 0 to 5 , and a score $\geq 3$ revealed high study quality.

Figure 1. Study flow diagram.

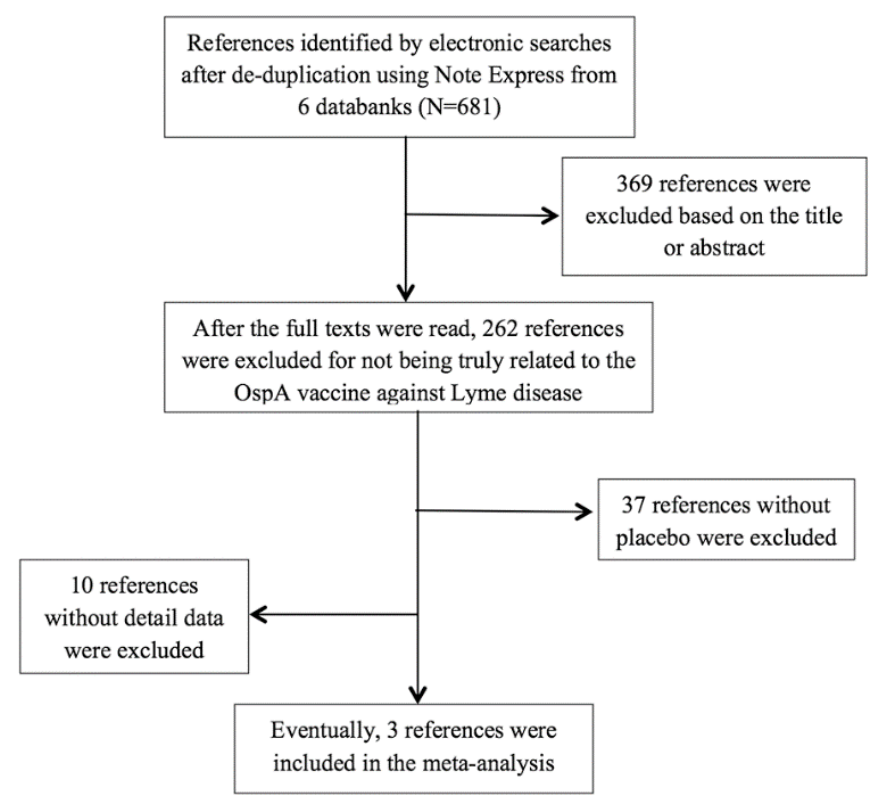




\section{Results}

After de-duplication, 681 studies of reference in the databases were found using the descriptor combinations previously established at the beginning of the research. A total of 369 references were excluded after abstract reading. Next, the full text of 312 articles was read and 262 references were excluded. A further 37 references were also excluded due to lack of placebo. The results were refined by excluding 10 studies [16,17,23-30] without detail data after more detailed analysis. The remaining 3 studies were included for meta-analysis [31-33]. The flow of study selection is shown in Figure 1.

\section{Immunogenicity and efficacy}

Efficacy and immunogenicity were explored by comparing IgG antibody titer of a high-dose group with low-dose group and the overall mean value of $\operatorname{IgG}$ antibody titer (data has been transformed using logarithm form) was calculated, as shown in Figure 2A. The heterogeneity test showed that $\mathrm{p}=0.000$ with I square of $99.5 \%$, meaning that there was heterogeneity among all the studies included, so the random-effect model should be applied for meta-analysis. The results of meta-analysis showed that the overall effect size was $6.39(95 \% \mathrm{CI}=3.87-8.90, \mathrm{p}=0.000)$, indicating that the overall effect size was statistically significant and that the higher the dose was, the higher the titer.

The funnel plot of immunogenicity of vaccine of OspA against Lyme disease (Figure 2B) shows that the studies were not quite symmetrically distributed and that a publication bias may exist; the Egger test was therefore used. The Egger plot in Figure 2C shows that the studies were within 95\% confidence interval, and the Egger test (Table 1) also shows that $p=0.963$, which is above 0.05 , meaning that there was no

Figure 2. Forest plot, funnel plot, and Egger plot of immunogenicity of vaccine of OspA against Lyme disease.
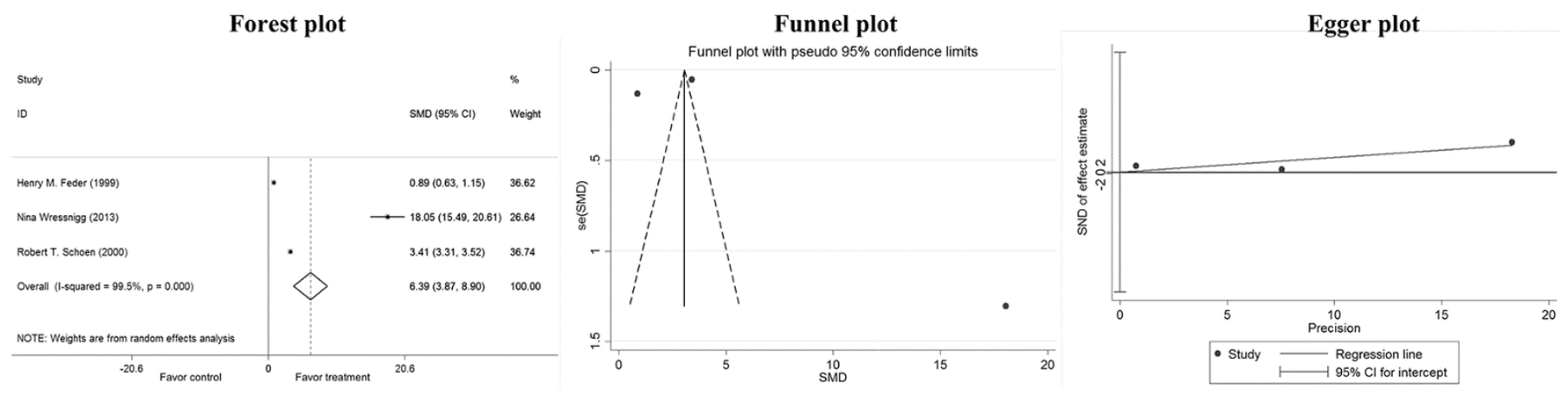

Table 1. Egger test of immunogenicity and side effects

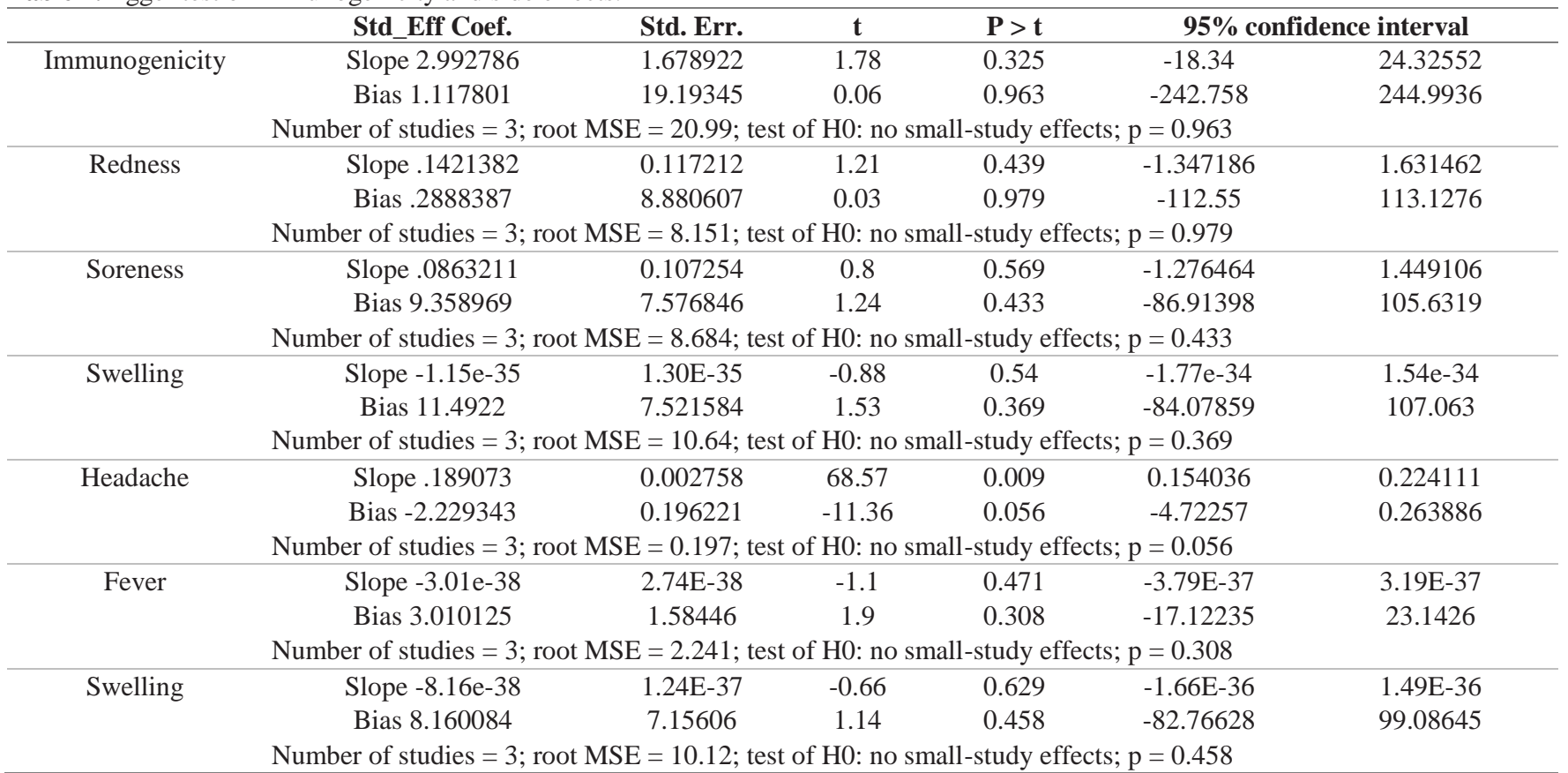


publication bias and that the results of the meta-analysis were reliable.

\section{Mean level of IgG titer}

A forest plot of immunogenicity of the OspA vaccine (Figure 3) was drawn. Because I square was 0\% with $\mathrm{p}$ value of the heterogeneity test of 0.996 , meaning that there was no heterogeneity among all the studies, the fixed-effect model was applied. The results of metaanalysis showed that overall effect size was 3.84 (95\% $\mathrm{CI}=3.64-4.04, \mathrm{p}=0.000)$, meaning that overall effect size (IgG titer) was truly above 0; furthermore, the geometric mean titer was $10^{\wedge} 3.84=6,918.31$, indicating that the OspA vaccine was effective and immunogenetic.

\section{Local side effects: Redness}

I square was $95.9 \%$ with $\mathrm{p}<0.05$, indicating heterogeneity, so the random-effect model was chosen for the meta-analysis. Results (Figure 4A) showed that the overall effect size was $0.17(95 \% \mathrm{CI}=-0.02-0.37$,
Figure 3. Forest plot of IgG antibody titer (logarithm) of vaccine of OspA against Lyme disease.

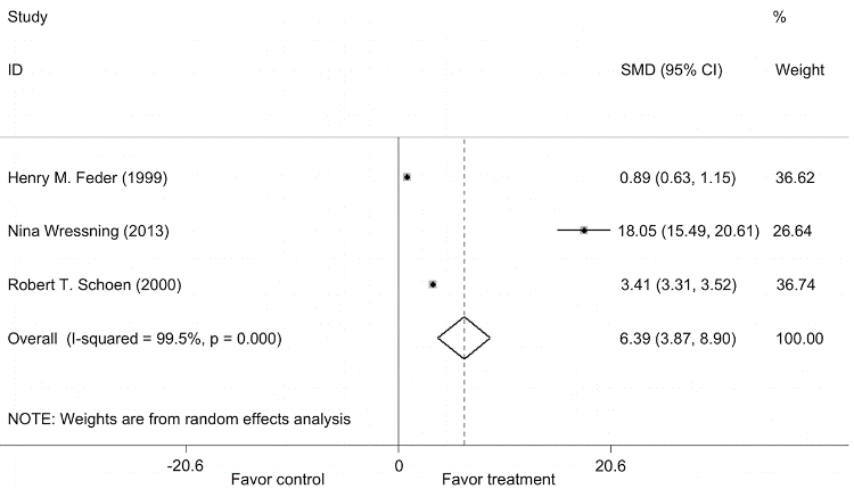

$\mathrm{p}=0.080)$. There was no difference between the incidence of redness with 0 , meaning that the local side effect of redness was at a low level. With respect to redness, the OspA vaccine is quite safe.

The funnel plot in Figure 4B shows that the studies were not quite symmetrically distributed, so a publication bias may exist, and the Egger test was

Figure 4. Forest plot, funnel plot, and Egger plot of local side effects.
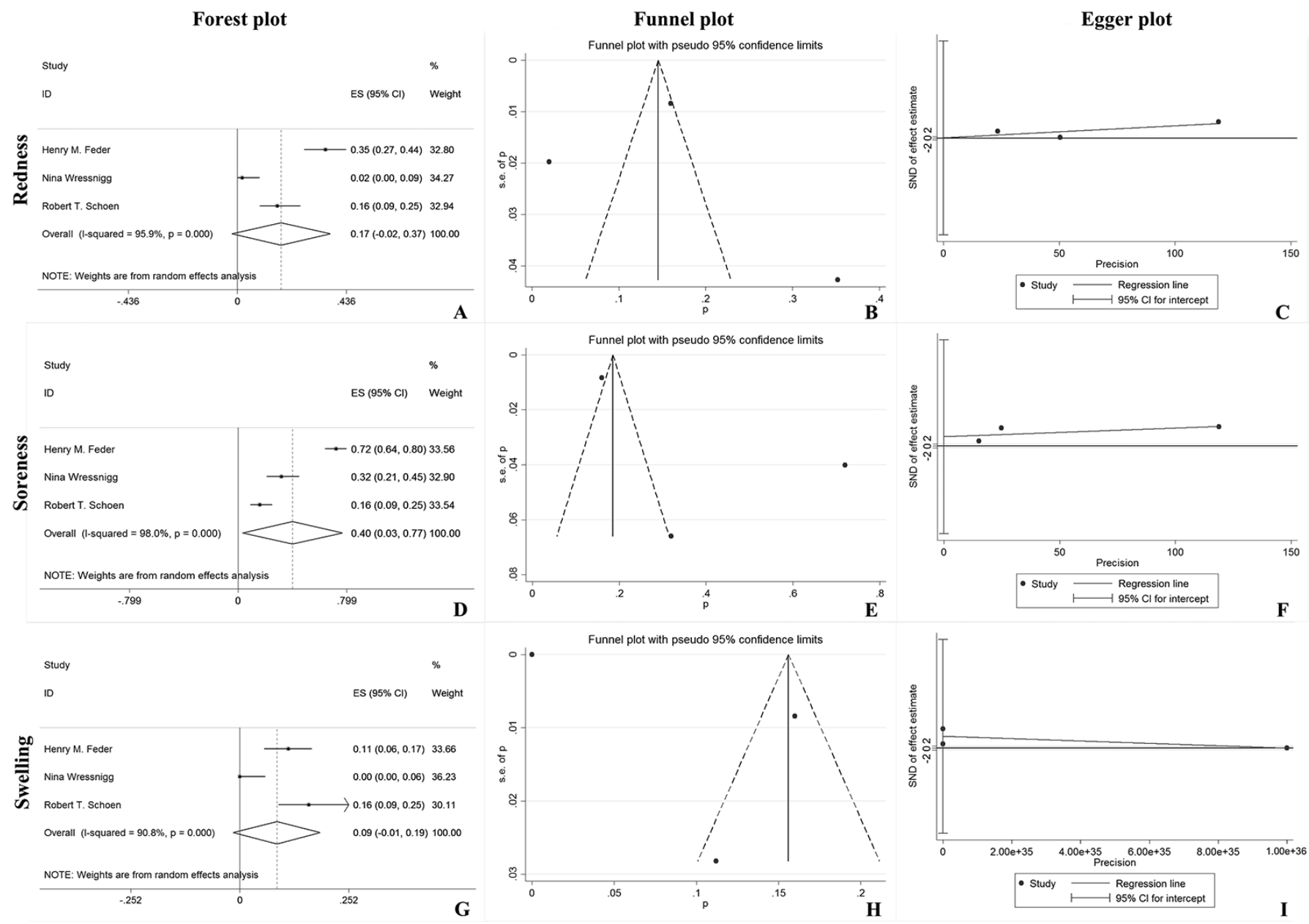
needed. The Egger plot of redness (Figure 4C) shows that the studies were all within $95 \%$ confidence interval; at the same time, the Egger test (Table 1) shows that $\mathrm{p}$ $=0.979$, so no publication bias existed and the conclusion of the forest plot was reliable.

\section{Local side effects: Soreness}

As heterogeneity existed $(\mathrm{p}=0.000$, I square $=$ 98\%), the random-effect model was applied for metaanalysis. Figure 4D shows that the overall effect size was $0.4(95 \% \mathrm{CI}=0.03-0.77, \mathrm{p}=0.033)$, meaning that incidence of soreness was different from 0 and the incidence of $40 \%$ was relatively high, indicating that the OspA vaccine is not satisfactory with respect to soreness.

The funnel plot in Figure 4E shows that the studies were not quite symmetrically distributed and that a publication bias should be further checked using the Egger test (Table 1). The Egger plot of soreness (Figure $4 \mathrm{~F})$ shows that all the studies were within $95 \%$ confidence interval; also, the Egger test in Table 1 shows that $\mathrm{p}=0.433$, so no publication bias existed and the conclusion of the forest plot was reliable.

\section{Local side effects: Swelling}

A forest plot was drawn (Figure 4G). As heterogeneity existed $(p=0.000$, I square $=90.8 \%)$, the random-effect model was applied for meta-analysis. Results showed that the overall effect size was 0.09 $(95 \% \mathrm{CI}=-0.01-0.19, \mathrm{p}=0.094)$. Because the $\mathrm{p}$ value was under 0.05 , the incidence of soreness was different from 0 and the incidence of $40 \%$ was relatively high, indicating that the OspA vaccine is not satisfactory with respect to swelling.

The funnel plot in Figure 4H shows that the studies were not quite symmetrically distributed and that a publication bias should be checked further using the Egger test (Table 1). The Egger plot in Figure 4I shows that all the studies were within $95 \%$ confidence interval, and the Egger test in Table 1 shows that $p=0.369$, so no publication bias existed and the conclusion of the forest plot was reliable.

Figure 5. Forest plot, funnel plot, and Egger plot of systemic side effects.
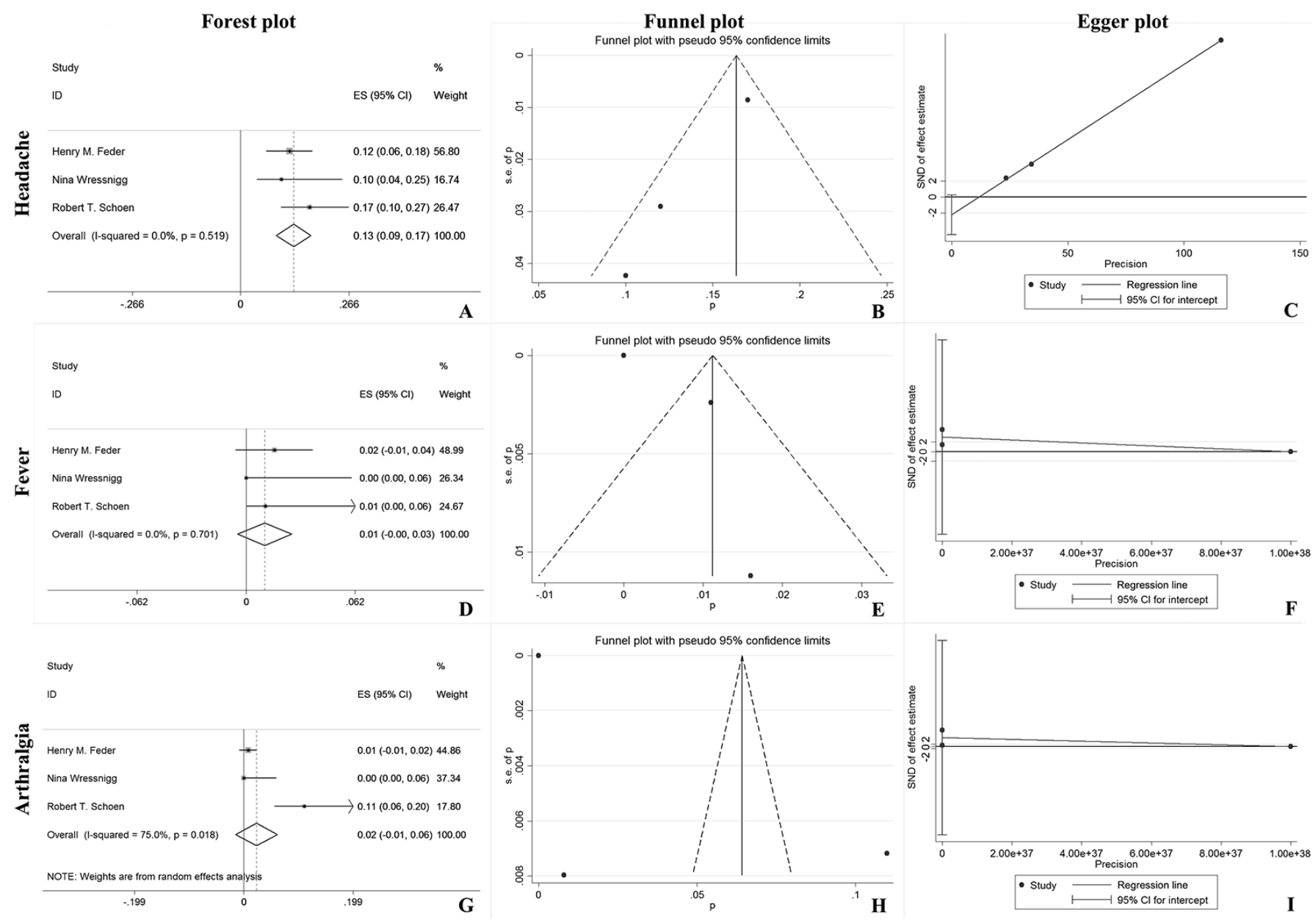
Systemic side effects: Headache

A heterogeneity test showed that $\mathrm{p}=0.519$ with I square of $0 \%$, indicating that there was no heterogeneity. The fixed-effect model was applied, and results (Figure 5A) showed that the overall effect size was $0.13(95 \% \mathrm{CI}=0.09-0.17, \mathrm{p}=0.000)$. Because the $\mathrm{p}$ value was under 0.05 , the incidence of headache was different from 0 but at a low level, indicating that the OspA vaccine is safe.

The funnel plot in Figure 5B shows that the studies were not quite symmetrically distributed and that a publication bias should be checked further using the Egger test (Table 1). The Egger plot in Figure 5C shows that all the studies were outside $95 \%$ confidence interval, but the Egger test in Table 1 shows that $\mathrm{p}=$ 0.056 , which is above 0.05 ; therefore, no publication bias existed and the conclusion of forest plot was reliable.

\section{Systemic side effects: Fever}

A heterogeneity test showed that $\mathrm{p}=0.701$ with I square of $0 \%$, indicating that there was no heterogeneity. The fixed-effect model was applied, and results (Figure 5D) show that the overall effect size was $0.01(95 \% \mathrm{CI}=-0.00-0.03, \mathrm{p}=0.179)$. Because $\mathrm{p}>$ 0.05 , the incidence of fever was equal to 0 , indicating that the OspA vaccine is safe.

The funnel plot in Figure 5E shows that the studies were not quite symmetrically distributed and one study was outside $95 \%$ confidence interval, so publication bias should be checked further using the Egger test (Table 1). The Egger plot in Figure 5F shows that all the studies were within $95 \%$ confidence interval, and the Egger test in Table 1 shows that $p=0.308$, which is above 0.05 ; therefore, no publication bias existed and the conclusion of the forest plot was reliable.

\section{Systemic side effects: Arthralgia}

A heterogeneity test showed that $\mathrm{p}=0.018$ with I square of $75 \%$, indicating that there was heterogeneity among the studies. The random-effect model was applied, and results (Figure 5G) show that the overall effect size was $0.02(95 \% \mathrm{CI}=-0.01-0.06, \mathrm{p}=0.230)$. Because $\mathrm{p}>0.05$, the incidence of arthralgia was equal to 0 , indicating that the OspA vaccine is safe.

The funnel plot in Figure 5H shows that the studies were not quite symmetrically distributed, and were all outside $95 \%$ confidence interval, so publication bias should be checked further using the Egger test (Table 1). The Egger plot in Figure 5I shows that all the studies were within $95 \%$ confidence interval, and the Egger test in Table 1 shows that $\mathrm{p}=0.458$, so no publication bias existed and the conclusion of the forest plot was reliable.

\section{Discussion}

We conducted this meta-analysis to provide a comprehensive and systemic evaluation of the OspA vaccine. The results of our meta-analysis found that the geometric mean titer of the OspA vaccine was 6,918.31, which is higher than that before injection, and that a higher dose of $30 \mu \mathrm{g}$ is accompanied by a higher titer than a lower dose of $15 \mu \mathrm{g}$, which was also found by Parenti et al. [34], indicating good immunogenicity and efficacy of the vaccine. We also found that incidence of local side effects (redness, soreness, and swelling) or systemic side effects (headache, fever, and arthralgia) occurred infrequently, which had no difference when compared with 0 except for soreness. The OspA vaccine against Lyme disease is therefore quite safe, as also reported by Poland et al. [28].

As the most common disease spread by ticks in the northern hemisphere, Lyme borreliosis has affected approximately 300,000 people each year in the United States and 65,000 people each year in Europe over the past decade [35-37]. In an environment with a growing number of Lyme borreliosis cases, vaccination is considered to be the most effective measure of controlling Lyme diseases and, in fact, is the only prevention method ever shown to be efficacious in large community trials [28].

Since Schaible et al. found that OspA could elicit protective immunity in 1990 [38], a series of studies focusing on OspA have been conducted. To summarize, the OspA vaccine has three main positive characteristics. First, it blocks transmission of Borrelia burgdorferi from ticks to the human body, and it is easier to test for efficacy. Second, as a subunit vaccine, it does not interfere with immunodiagnosis. Third, the vaccine targets a reasonably conserved protein within the species [8]. Though there are some prospective Lyme candidate vaccine antigens from Borrelia burgdorferi, such as OspA, OspB, OspC, DbpA, and Bbk32(p35), only OspA is approved by the Food and Drug Administration (FDA) to be a transmissionblocking vaccine. LYMErix, marketed in the United States in 1999, was the first OspA-based human vaccine approved by the FDA. However, multiple reasons led to its demise. This was the first modern case of a FDAlisted vaccine being withdrawn because of low needs and litigation [39]. Another OspA-oriented vaccine that Pasteur Merieux-Connaught developed never came into market, although its productive forces were equivalent 
or even superior to those of LYMErix [17]. The loss of LYMErix invigorated examinations concerning further development of Lyme borreliosis vaccines.

Recently, improvements have been made on a newgeneration, multivalent OspA-based Lyme borreliosis vaccine intended to protect against all Borrelia burgdorferi sensu lato strains related to global human diseases. With reference to preclinical research, antibody reactions were induced by a single recombinant OspA including protective components from two OspA serotypes (1 and 2), and thus secured mice from being infected with Borrelia burgdorferi (OspA-1) or Borrelia afzelii (OspA-2) [39]. Research concerning the multivalent recombinant OspA vaccine showed that immunized mice were protected from being infected with Borrelia burgdorferi, B. afzelii, Borrelia bavariensis, and Borrelia garinii. Likewise, the six OspA types targeted by the vaccine not only induced practical antibodies, but different types of Borrelia, encompassing B. spielmani, $B$. valaisiania, $B$. lusitaniae, and B. japonica, which further demonstrated that the vaccine could possibly protect against Lyme borreliosis comprehensively. The preparatory information shows that the new multivalent chimeric OspA vaccine could potentially be an imperative strategy to alleviate the developing effect of this debilitating illness.

To strengthen the quality of this meta-analysis, a thorough literature search was performed in different databases. The literature selection and quality assessment were carried out strictly in accordance with the well-defined inclusion and exclusion criteria to reduce the selection bias and confounding bias, so some important works had to be excluded [16,17]. In the process of statistical analysis, the heterogeneity test and publication bias were important.

Nevertheless, limitations of this meta-analysis should be acknowledged. Firstly, we found heterogeneity in redness, soreness, swelling, and arthralgia across studies. However, we were not able to determine the source of the heterogeneity. Secondly, since we only searched papers published in English and Chinese and all included trails were published in English, a language bias may exist. Thirdly, the possibility of selection bias may exist because unpublished related studies were not included. More high-quality research is needed for more reliable conclusions.

\section{Conclusions}

Despite the limitations mentioned above, the results of our meta-analysis found that the OspA vaccine against Lyme disease is safe and has good immunogenicity and efficacy. Instead of stagnating or giving up, further research on improving the vaccine is needed. On the foundation of preliminary studies, we can attempt to develop new vaccines for human use.

\section{Acknowledgements}

This work was supported by National Natural Science Foundation of China grants (No. 81560596, No. 81060134, No. 31560051, and No. 81560596), and Natural Foundation of Yunnan Province grants (No. 2012FB011, No. 2013FZ05, No. 2014FA011, and No. 2014FB001), but funding institutions had no role in the study design or review of the manuscript. Yunnan Province Key Laboratory for Tropical Infectious Diseases in Universities, Kunming, China, Yunnan Province Integrative Innovation Center for Public Health, Diseases Prevention and Control, Kunming Medical University, Kunming, China, Yunnan Demonstration Base of International Science and Technology Cooperation for Tropical Diseases, Kunming, China also supported this study. We would like to acknowledge the helpful comments on this paper received from our reviewers.

\section{Authors' contributions}

Hua Zhao and Fukai Bao conceived the idea, designed the study, and wrote the manuscript. All authors contributed to the qualitative and quantitative synthesis of the included trials. Aihua Liu and Fukai Bao revised the manuscript. All authors read and approved the final version of the manuscript.

\section{References}

1. Stanek G, Strle F (2004) Lyme borreliosis. Lancet Infect Dis 4: 197-198.

2. Steere AC, Livey I (2012) Lyme disease vaccines. In Plotkin SA, Orenstein WA, Offit PA, editors. Vaccines, 6th edition. Philadelphia: Elsevier: 1122-1132.

3. World Health Organization (2012) Lyme Borreliosis (Lyme disease). Available: http://www.who.int/ith/diseases/Lyme/en. Accessed 11 June 2015.

4. Stanek G, Fingerle V, Hunfeld KP, Jaulhac B, Kaiser R, Krause A, Kristoferitsch W, Oconnell S, Ornstein K, Strle F (2011) Lyme borreliosis: clinical case definitions for diagnosis and management in Europe. Clin Microbiol Infect 17:69-79.

5. Kolb P, Vorreiter J, Habicht J, Bentrop D, Wallich R, Nassal M (2014) Soluble cysteine-rich tick saliva proteins Salp15 and Iric-1 from E. coli. Febs Open Bio 5: 42-55.

6. Small CM, Ajithdoss DK, Aline RH, Waithaka M, EsteveGassent MD (2014) Immunization with a Borrelia burgdorferi BB0172-derived peptide protects mice against Lyme disease. Plos One 9: e88245-e88245.

7. Bhattacharya D, Bensaci M, Luker KE, Luker G, Wisdom S, Telford SR, Hu LT (2011) Development of a baited oral vaccine for use in reservoir-targeted strategies against Lyme disease. Vaccine 29: 7818-7825.

8. Embers ME, Narasimhan S (2013) Vaccination against Lyme disease: past, present, and future. Front Cell Infect Microbiol 3: 6. 
9. Lafleur RL, Callister SM, Dant JC, Jobe DA, Lovrich SD, Warner TF, Wasmoen TL, Schell RF (2010) One-year duration of immunity induced by vaccination with a canine Lyme disease bacterin. Clin Vaccine Immunol 17: 870-874.

10. Livey I, O'Rourke M, Traweger A, Savidis-Dacho H, Crowe BA, Barrett PN, Yang X, Dunn JJ, Luft BJ (2011) A new approach to a Lyme disease vaccine. Clin Infect Dis 52 Suppl 3: 266-270.

11. Meirelles Richer L, Aroso M, Contente-Cuomo T, Ivanova L, Gomes-Solecki M (2011) Reservoir targeted vaccine for Lyme borreliosis induces a yearlong, neutralizing antibody response to OspA in white-footed mice. Clin Vaccine Immunol 18: 1809-1816.

12. Voordouw MJ, Tupper H, Önder Ö, Devevey G, Graves CJ, Kemps BD, Brisson D (2013) Reductions in human Lyme disease risk due to the effects of oral vaccination on tick-tomouse and mouse-to-tick transmission. Vector Borne Zoonotic Dis 13: 203-214.

13. Fikrig E, Barthold SW, Kantor FS, Flavell RA (1990) Protection of mice against the Lyme disease agent by immunizing with recombinant OspA. Science 250: 553-556.

14. Fikrig E, Barthold SW, Kantor FS, Flavell RA (1992) Longterm protection of mice from Lyme disease by vaccination with OspA. Infect Immun. 60: 773-777.

15. Fikrig E, Telford SR, Barthold SW, Kantor FS, Spielman A, Flavell RA (1992) Elimination of Borrelia burgdorferi from vector ticks feeding on OspA-immunized mice. PNAS 89: 5418-5421.

16. 16. Steere AC, Sikand VK, Meurice F, Parenti DL, Fikrig E, Schoen RT, Nowakowski J, Schmid CH, Laukamp S, Buscarino C, Krause DS (1998) Vaccination against Lyme disease with recombinant Borrelia burgdorferi outersurface lipoprotein A with adjuvant. Lyme Disease Vaccine Study Group. N Engl J Med 339: 209-215.

17. Sigal LH, Zahradnik JM, Lavin P, Patella SJ, Bryant G, Haselby R, Hilton E, Kunkel M, Adler-Klein D, Doherty T, Evans J, Molloy PJ, Seidner AL, Sabetta JR, Simon HJ, Klempner MS, Mays J, Marks D, Malawista SE (1998) A vaccine consisting of recombinant Borrelia burgdorferi outersurface protein A to prevent Lyme disease. Recombinant Outer-Surface Protein A Lyme Disease Vaccine Study Consortium. N Engl J Med 339: 216-222.

18. Harris RJ, Bradburn MJ, Deeks JJ, Harbord RM, Altman DG, Sterne JAC (2008) metan: fixed- and random-effects metaanalysis. Stata J 8: 3-28.

19. Egger M, Smith GD, Schneider M, Mnder C (1997) Bias in meta-analysis detected by a simple, graphical test. BMJ 315 : 629-634.

20. Higgins JPT, Green S (2011) Cochrane Handbook for Systematic Reviews of Interventions Version 5.1.0. The Cochrane Collaboration. Available: http://www.cochrane handbook.org. Accessed 11 June 2015.

21. Higgins JP, Thompson SG, Deeks JJ, Altman DG (2003) Measuring inconsistency in meta-analyses. BMJ 327: 557-560.

22. Jadad AR, Moore RA, Carroll D, Jenkinson C, Reynolds DJM, Gavaghan DJ, Mcquay HJ (1996) Assessing the quality of reports of randomized clinical trials: is blinding necessary? Controlled Clin Trials 17: 1-12.

23. Steere AC, Levin RE, Molloy PJ, Kalish RA, Abraham JR, Liu NY, Schmid CH (1994) Treatment of Lyme arthritis. Arthritis Rheum 37: 878-888.

24. Wormser GP, Nowakowski J, Nadelman RB, Schwartz I, Mckenna D, Holmgren D, Aguero-Rosenfeld M (1998)
Efficacy of an OspA vaccine preparation for prevention of Lyme disease in New York State. Infection 26: 208-212.

25. Van HC, Lebacq E, Beran J, Parenti D (1999) Alternative vaccination schedules $(0,1$, and 6 months versus 0,1 , and 12 months) for a recombinant OspA Lyme disease vaccine. Clin Infect Dis 28: 1260-1264.

26. Simon MM, Bauer Y, Zhong W, Hofmann H, Wallich R (1999) Lyme disease: pathogenesis and vaccine development. Zentralbl Bakteriol 289: 690-695.

27. Luke CJ, Marshall MA, Zahradnik JM, Bybel M, Menefee BE, Barbour AG (2000) Growth-inhibiting antibody responses of humans vaccinated with recombinant outer surface protein A or infected with Borrelia burgdorferi or both. J Infect Dis 181: 1062-1068.

28. Poland GA, Jacobson RM (2001) The prevention of Lyme disease with vaccine. Vaccine 19: 2303-2308.

29. Wressnigg N, Barrett PN, Pöllabauer EM, O'Rourke M, Portsmouth D, Schwendinger MG, Crowe BA, Livey I, Dvorak T, Schmitt B, Zeitlinger M, Kollaritsch H, Esen M, Kremsner PG, Jelinek T, Aschoff R, Weisser R, Naudts IF, Aichinger G (2014) A Novel multivalent OspA vaccine against Lyme borreliosis is safe and immunogenic in an adult population previously infected with Borrelia burgdorferi sensu lato. Clin Vaccine Immunol 21: 1490-1499.

30. Lindgren E (2001) Lyme borreliosis in Europe: influences of climate and climate change, epidemiology, ecology and adaptation measures. Geneva: World Health Organization. 728.

31. Feder HJ, Beran J, Van Hoecke C, Abraham B, De Clercq N, Buscarino C, Parenti DL (1999) Immunogenicity of a recombinant Borrelia burgdorferi outer surface protein A vaccine against Lyme disease in children. J Pediatr 135: 575579.

32. Wressnigg N, Pöllabauer EM, Aichinger G, Portsmouth D, Löw-Baselli A, Fritsch S, Livey I, Crowe BA, Schwendinger M, Brühl P, Pilz A, Dvorak T, Singer J, Firth C, Luft B, Schmitt B, Zeitlinger M, Müller M, Kollaritsch H, Paulke-Korinek M, Esen M, Kremsner PG, Ehrlich HJ, Barrett PN (2013) Safety and immunogenicity of a novel multivalent OspA vaccine against Lyme borreliosis in healthy adults: a double-blind, randomised, dose-escalation phase 1/2 trial. Lancet Infect Dis 13: 680-689.

33. Schoen RT, Sikand VK, Caldwell MC, Van Hoecke C, Gillet M, Buscarino C, Parenti DL (2000) Safety and immunogenicity profile of a recombinant outer-surface protein a Lyme disease vaccine: Clinical trial of a 3 -dose schedule at 0,1 , and 2 months. Clin Ther 22: 315-325.

34. Parenti DL, Gillet M, Sennewald E (1998) Correlate of protection for Lyme disease (LD) using LYMErixTM, recombinant, adjuvanted Borrelia burgdorferi outer surface lipoprotein A (LOspA) vaccine. Infectious Diseases Society of America Annual Meeting. 208.

35. Defense Pest Management Information Analysis Center (2001) Regional disease vector ecology profile central Europe (serial report). Armed Forces Pest Management Board, Forest Glen Section. Washington: Walter Read Army Medical Center. 134136.

36. Shapiro ED (2014) Clinical practice. Lyme disease. N Engl J Med 370: 1724-1731.

37. Berger SA (2014) Lyme Disease: Global Status. Gideon ebooks: $7 . \quad$ Available: http://www.gideononline.com/ebooks/disease/Lyme-diseaseglobal-status. Accessed 11 June 2015. 
38. Schaible UE, Kramer MD, Eichmann K, Modolell M, Museteanu C, Simon MM (1990) Monoclonal antibodies specific for the outer surface protein A (OspA) of Borrelia burgdorferi prevent Lyme borreliosis in severe combined immunodeficiency (scid) mice. Proc Natl Acad Sci U S A 87: 3768-3772.

39. Barrett PN, Portsmouth D (2013) The need for a new vaccine against Lyme borreliosis. Expert Rev Vaccines 12: 101-103.

\section{Corresponding author}

Fukai Bao, MD, PhD

Department of Microbiology and immunology/The Institute for Tropical Medicine Kunming Medical University

Renmingxi road, 1186, 650500 Kunming

Yunnan, P. R. of China

Phone: +86 13888369882

Fax: 0086-871-65922857

Email: baofukai@126.com, baofukai@kmmu.edu.cn

Conflict of interests: No conflict of interests is declared. 